\title{
Legumes for South Island tussock grassland environments - an evaluation of plant survival and growth at some inland Otago and Canterbury trials
}

\author{
B. WILLS ${ }^{1}, \mathrm{~K}$. TRAINOR ${ }^{2}$ and D. SCOTT ${ }^{1}$ \\ ${ }^{1}$ AgResearch, PO Box 228, Alexandra \\ ${ }^{2} P O$ Box 115, Lake Tekapo \\ barrie.wills@agresearch.co.nz
}

\begin{abstract}
Many legumes have been trialled for establishment, persistence and productivity in the tussock grassland region of the South Island, New Zealand. This paper presents information on long-term persistence and growth based on a survey of surviving legume species from 45 previous plant accession trials, established over a range of sites and environments (predominantly dryland) throughout Otago and Canterbury. Sites were visited in January/February 2003 and identified plants were visually scored for several growth-related parameters. Most sites were open to stock grazing and all were affected by drought during the current growing season.

Of the legume species that seed is not commercially available for in New Zealand, the most persistent and vigorous were: Lupinus polyphyllus, Trifolium medium, Coronilla varia, Dorycnium hirsutum, Melilotus alba and M. officinalis, Astragalus falcatus, T. canescens, D. pentaphyllum, and A. cicer. These plants may usefully supplement tussock grassland vegetation under environmental and management conditions including periodic drought and the moderate to light grazing experienced at most of the trial sites visited. This paper reviews growth and survival of those legumes, and provides a guide to species with potential for revegetation in dryland and semi-arid environments that warrant further research into site $\mathrm{x}$ management interactions and possibly commercial development.
\end{abstract}

Key words: legume persistence, Otago/Canterbury, revegetation, tussock grassland

\section{Introduction}

The objective was to survey legume species across a range of (mainly) mid-altitude hill country trial sites in Otago and Canterbury, particularly those within drier environments, and identify those that have persisted and/or grew well. Those legumes may then be considered for inclusion in tussock grassland revegetation programmes, or to assist with pastoral improvement when management and site factors are further defined. Most trials pre-date the change to Foundation for Research, Science and Technology based research funding and, although many have been discontinued, the information available from them is still considered relevant to pastoral farming given their relative longevity and, in many cases, exposure to 'normal' grazing management practice.

Research trials including legumes were initiated in the early 1900s in the Mackenzie Basin and Central Otago and have been described in the surveys and reports of McPherson, McGillivray, Cockayne, Calder, Zotov and others, documented chiefly in early volumes of the New Zealand Journal of Agriculture. These included single species from seed or plants, and some trials with multiple species mixtures.

During the 1980s and 1990s, on-farm agricultural and soil conservation research and technology transfer was conducted by the former Ministry of Agriculture \& Fisheries, Department of Scientific \& Industrial Research, and Ministry of Works \& Development Water \& Soil Division, now largely combined into AgResearch. Various reviews and papers relating to this plant introduction work have been published; Wills (1984), Scott et al. (1995), van Kraayenoord \& Hathaway (1986), Chapman et al. (1989), Woodman et al. (1992, 1996), Douglas et al. (1996) and Scott (2001).

Comprehensive technical documentation was also distributed in association with the numerous MRDC/ MAF/AgR field days held at properties like Belfield (Hakataramea Valley, 1991), Glenfoyle, Ardlui, Earnscleugh (Central Otago, 1992) and North Otago (1992). This paper provides updated information for several of these trials and an indication of future perspective relating to possible legume application based consideration of current and historical results. 


\section{Methods}

Seventeen sites in Central Otago (schistose base material) and the Mackenzie Basin (greywacke) were visited in January/February 2003. These were comprised of 45 separate trial plots including Central Otago: Bendigo (five plots), Galloway (two), Earnscleugh-Omeo (three), Earnscleugh-Shepherds Flat (four), Northburn-Cockayne (10); and Mackenzie/ Waitaki: Haka-Belfield (one), Otematata (three), Stony Creek (one), Black Forest (one), Haldon Airstrip (one), Sievwright-Holbrook (one), Tara Hills (four), Glentanner (one), Ben Ohau (one), Ruataniwha (one), Wolds (one), Mt John (several). Site location and 30 year normal rainfall data (Tomlinson \& Sansom 1994) for the nearest meteorological stations are detailed in Table 1.

Table 1 New Zealand Map Series 260 site references and long term normal annual rainfall $(\mathrm{mm})$ of nearest meteorological station.

\begin{tabular}{lcc}
\hline Site & Reference & Rainfall (Site) \\
\hline Bendigo & G41 223801 & 401 (Cromwell) \\
Mid-Galloway & G42 388434 & 412 (Clyde) \\
Top-Galloway & G42 433338 & 597 (Blackstone Hill) \\
Earnscleugh-Omeo & G42 178453 & 412 (Clyde) \\
Earnscleugh-Shepherds Flat & G42 145484 & 412 (Clyde) \\
Northburn-Cockayne (Top plot) & G41 162672 & 401 (Cromwell) \\
Haka-Belfield & I42 139113 & 535 (Waitaki) \\
Otematata & H40 860159 & 535 (Waitaki) \\
Stony Creek & I39 614414 & 646 (Twizel) \\
Black Forest & I39 949371 & 646 (Twizel) \\
Haldon Airstrip & I39 922469 & 646 (Twizel) \\
Sievwright-Holbrook & I38 118781 & 646 (Twizel) \\
Tara Hills (Red Flat) & H39 622264 & 546 (Tara Hills) \\
Tara Hills (hill) & I39 641246 & 546 (Tara Hills) \\
Glentanner (top) & H37 764962 & 854 (Braemar) \\
Ben Ohau & H38 821586 & 646 (Twizel) \\
Ruataniwha & H38 744581 & 646 (Twizel) \\
Wolds & I38 979758 & 597 (Tekapo) \\
Mt John & 597 (Tekapo) \\
\hline
\end{tabular}

The majority of trial plots ranged in size from 0.25-1.0 ha. although some, like Bendigo and Mt John, covered several hectares. Earnscleugh, Northburn and Glentanner sites comprise several small plots totalling 2-5 ha. They cover a wide range of altitude, from 300-1 $200 \mathrm{~m}$ above sea level with most plots in the 400-700 $\mathrm{m}$ range and receive 400-650 $\mathrm{mm}$ annual rainfall (Table 1). The high Glentanner sites were the exception with $850 \mathrm{~mm}$ annual rainfall, but it was considered expedient to include them in the survey due to their proximity to other Mackenzie Basin trials.

Approximately $40 \%$ of the trial species surveyed were drilled or oversown, the remainder being handplanted, thus a range of establishment technique was represented. Site maintenance and management had changed considerably since the original trials were evaluated. Fences were either down or removed with only a few sites left intact and ungrazed (specifically the Sievwright, Black Forest, Haldon, and one high altitude Glentanner, plots). All others had some degree of grazing, either recent or historic, with open access to stock (and rabbits) from adjacent farm blocks. Grazing and fertility over the past decade or more have been determined by standard management of those farm blocks and, although not necessarily ideal for the legume species, it now represents a 'real world' situation.

At the assessment in 2002/ 2003, most sites were unstocked (since at least December 2002) and few rabbits were noted. Exceptions included Ben Ohau (rabbit numbers were high and $T$. arvense was dominant), Ruataniwha (Merino sheep were grazing in the vicinity), and Earnscleugh-Shepherds Flat (cattle had access from paddocks enclosing the trial plots). Part of one plot at Otematata was open and had been grazed recently. Most sites contained several species with a range of palatability. All were well represented in the survey results (Tables 3,4), not just those normally considered 'less palatable' as 
might be expected if sites had been heavily and/or recently grazed.

At each site, a record was made of the legume species present, and the height and spread of representative plants was measured. For uniformity of assessment across differing sites, plant vigour, survival and productivity were visually scored. Scoring was done on a '0' to ' 3 ' basis (modified from Haydock \& Shaw 1975). A '0' score indicated plants were not growing (low vigour), there were few present (poor survival), or little foliage was present (low productivity). Only extremely vigorous (growing actively at time of assessment), numerous (based on original plantings or seeding) or productive (having accumulated substantial forage, not necessarily fresh) plants recorded a maximum score of ' 3 ' in each category. Limited plant growth caused by the dry seasonal conditions precluded effective use of a wider scoring range (eg. 0-5). aspect plot was divided into $2 \mathrm{~m}$ wide strips that were traversed, noting plant numbers for identifiable legume species. Plant numbers were totalled for each replicate and standard errors of the means were calculated for this data. Legume plant height and spread were also measured and those data were combined with plant number data to give an approximation of the percent plant groundcover and plant foliar volume. A similar calculation was also carried out for hard tussock, Festuca novaezealandiae, as a grassland comparison. This was based on the height and spread of typical local stands of hard tussock, which had a density of about 1 plant/ $\mathrm{m}^{2}$.

Due to the considerable variation in site, species, environment and management, statistical analysis (standard errors of the mean) was only carried out for data from the replicated Earnscleugh - Shepherds Flat, sunny aspect plot.

Table 2 Monthly rainfall (mm) at Clyde, Tara Hills and Tekapo Airport, July 2002 to May 2003.

\begin{tabular}{lcccccccccccc}
\hline Month & Jul & Aug & Sep & Oct & Nov & Dec & Jan & Feb & Mar & Apr & May & Total \\
\hline Clyde & 6 & 36 & 29 & 24 & 36 & 41 & 57 & 19 & 9 & 16 & 25 & 298 \\
Tara & 22 & 37 & 55 & 5 & 36 & 38 & 46 & 34 & 10 & 11 & 54 & 318 \\
Tekapo & 28 & 57 & 96 & 23 & 51 & 41 & 35 & 39 & 20 & 13 & 83 & 486 \\
\hline
\end{tabular}

Each species plot, group or area within a trial site was ranked for vigour, survival and productivity. If replication was evident, sub-plots were scored separately and a site mean was obtained. This information was entered into a spreadsheet and sorted by the appropriate parameter (vigour or survival or productivity, or a combination) prior to tabulation. Height and spread data were not used in the sorting.

An 'accelerated screening trial', designed to evaluate 'persistence attributes' in a wide range of grasses and legumes, was established in 1992 at Earnscleugh Station (Fraser 1992), Tara Hills and Mt John. This only remained intact at Earnscleugh Station Shepherds Flat where stock (cattle) had access to the sunny, shady and neutral aspect plots. While it was not possible to accurately define the original pegged plot layout, the four replicates within each trial were still identifiable. The sunny aspect plot there contained several legume species, thus a more detailed survey of it was justified to define long-term plant survival and growth.

Each replicate (approximately $0.1 \mathrm{ha}$ ) in the sunny

\section{Results}

\section{Precipitation}

Monthly and total rainfall records for Clyde, Tara Hills and Tekapo Airport during the past growing season are presented in Table 2 . They provide an indication of seasonal precipitation, which can be compared to the 30-year normal records for selected regional stations (Table 1).

\section{Legume survival}

Across all trial sites, nine genera recorded the highest survival ranking of ' 3 '. These were represented by some 15 species (Table 3 ), the majority of those being from the genus Trifolium with five species (including T. ambiguum, T. arvense, T. hybridum, T. medium and T. repens) growing at several sites. Although these plants were all characterised by high survival, their rankings for vigour and productivity were variable. Sites with a high survival ranking for two or more legume species included Bendigo, both Earnscleugh sites (Omeo and Shepherds Flat), Mt John, Stony Ck, Glentanner (lower) and mid-Galloway. 
Table 3 Site, altitude, age and establishment method of legume species that recorded the highest survival ranking (3) from trial plots assessed in 2003.

\begin{tabular}{|c|c|c|c|c|}
\hline Establishment site & Altitude $(\mathrm{m})$ & Years established & Binomial & Method \\
\hline Ben Ohau -Acheron Flats & 450 & $25-30$ & Trifolium arvense & $\mathrm{R}$ \\
\hline \multirow[t]{2}{*}{ Bendigo } & 390 & $17-27$ & Dorycnium hirsutum & $\mathrm{D}$ \\
\hline & & & D. hirsutum & $P, D$ \\
\hline Earnscleugh AccScr Flat & 600 & $10-11$ & T. arvense & $\mathrm{R}$ \\
\hline \multirow[t]{4}{*}{ Earnscleugh AccScr Sunny } & 600 & $10-11$ & Coronilla varia & $\mathrm{P}$ \\
\hline & & & Lotus corniculatus & $\mathrm{P}$ \\
\hline & & & T. arvense & $\mathrm{R}$ \\
\hline & & & T. repens & $\mathrm{P}$ \\
\hline \multirow[t]{3}{*}{ Earnscleugh AccScr Shady } & 590 & $10-11$ & T. ambiguum & $\mathrm{P}$ \\
\hline & & & T. arvense & $\mathrm{R}$ \\
\hline & & & T. repens & $\mathrm{P}$ \\
\hline \multirow[t]{7}{*}{ Earnscleugh-Omeo } & 270 & $10-11$ & Astragalus cicer & $\mathrm{P}$ \\
\hline & & & A. falcatus & $\mathrm{P}$ \\
\hline & & & D. hirsutum & $P, D$ \\
\hline & & & D. pentaphyllum & $P, D$ \\
\hline & & & Melilotus alba & $\mathrm{P}$ \\
\hline & & & M. officinalis & $\mathrm{P}$ \\
\hline & & & T. ambiguum & $\mathrm{P}$ \\
\hline \multirow[t]{2}{*}{ Glentanner (Lower Chalet) } & 840 & $28-30$ & L. corniculatus & $\mathrm{P}, \mathrm{O} / \mathrm{S}$ \\
\hline & & & Lupinus polyphyllus & $S$ \\
\hline \multirow[t]{2}{*}{ Mid-Galloway } & 500 & $10-20$ & D. hirsutum & $\mathrm{O} / \mathrm{S}$ \\
\hline & & & T. ambiguum & $\mathrm{O} / \mathrm{S}$ \\
\hline \multirow[t]{4}{*}{ Mt John } & 760 & $8-12$ & Lotus corniculatus & $\mathrm{P}, \mathrm{O} / \mathrm{S}$ \\
\hline & & & Lupinus polyphyllus & $\mathrm{P}, \mathrm{O} / \mathrm{S}$ \\
\hline & & & T. arvense & $\mathrm{R}$ \\
\hline & & & T. medium & $\mathrm{P}, \mathrm{O} / \mathrm{S}$ \\
\hline \multirow[t]{2}{*}{ Northburn-Cockayne } & 600 & 80 & Medicago sativa & $\mathrm{O} / \mathrm{S}$ \\
\hline & & & T. medium & $\mathrm{P}$ \\
\hline Otematata & 490 & $20-25$ & D. pentaphyllum & $\mathrm{P}, \mathrm{O} / \mathrm{S}$ \\
\hline Sievwright-Holbrook & 670 & 60 & T. medium & $\mathrm{P}$ \\
\hline \multirow[t]{3}{*}{ Stony Creek } & 750 & 19 & A.falcatus & $\mathrm{P}$ \\
\hline & & & D. hirsutum & $\mathrm{P}$ \\
\hline & & & D. pentaphyllum & $P$ \\
\hline Tara Hills-Keoghan & 520 & 20 & T. arvense & $\mathrm{R}$ \\
\hline Top-Galloway & 790 & $20-30$ & T. ambiguum & $\mathrm{O} / \mathrm{S}$ \\
\hline \multirow[t]{2}{*}{ Wolds } & 640 & $30-35$ & T. hybridum & $\mathrm{O} / \mathrm{S}$ \\
\hline & & & T. repens & $\mathrm{O} / \mathrm{S}$ \\
\hline
\end{tabular}

AccScr $=$ Accelerated Screening trial. Establishment method includes $\mathrm{D}=$ drilling, $\mathrm{O} / \mathrm{S}=$ oversown/seeded, $\mathrm{P}=$ hand planted, $R=$ resident. NB. vigour and productivity rankings varied (compare Table 5). 
Table 4 Failed legume species with identified establishment method(s) and reason(s) for failure (where known).

\begin{tabular}{|c|c|c|c|c|}
\hline Binomial & Site & rs Established & Method & Comments \\
\hline Astragalus cicer & Bendigo & $1976-86$ & Drilled & Seed germination failed \\
\hline A. cicer & Bendigo & $1976-86$ & Plants & Failed after 2-3 yrs, drought \\
\hline A. cicer & Top-Galloway & 1983-93 & Plants, drilled & Seed germination failed, cold temperatures \\
\hline A. falcatus & Top-Galloway & 1983-93 & Plants, drilled & Seed germination failed, cold temperatures \\
\hline A. glycyphyllos & Top-Galloway & 1983-93 & Drilled & Seed germination failed, cold temperatures \\
\hline Dorycnium rectum & Bendigo & $1976-86$ & Plants & Drought failure \\
\hline D. rectum & Top-Galloway & $1983-93$ & Seed & Seed germination failed \\
\hline D. rectum & Otematata & 1978-93 & Plants & Drought stressed, frosting? \\
\hline D. rectum & Stony Creek & 1991-92 & Plants & Frost failure? \\
\hline D. suffruticosum & Bendigo & $1976-86$ & Plants & Drought failure \\
\hline Hedysarum coronariu & Bendigo & 1976-86 & Drilled & Seed germination failed \\
\hline H. coronarium & Bendigo & $1976-86$ & Plants, seed & Drought failure \\
\hline H. coronarium & Otematata & $1978-93$ & O/S seed & Drought and frosting failure \\
\hline Lotus caucasicus & Top-Galloway & 1983-93 & Drilled & Seed germination failed \\
\hline L. corniculatus & Acheron Flats & 1973-78 & O/S Seed & Originally L. cornic. dominant, many rabbits \\
\hline L. corniculatus & Ruataniwha & $1975-82$ & O/S Seed & Originally L. cornic. dominant, now vernal. \\
\hline L. corniculatus & Wolds & 1968-73 & Plants, seed? & From KF O'Connor trial, not identified \\
\hline L. tenuis & Bendigo & 1976-86 & Drilled & Failed after 2-3 yrs, drought \\
\hline Lupinus polyphyllus & Bendigo & $1976-86$ & Drilled & Failed after $1 \mathrm{st}$ yr, drought \\
\hline L. polyphyllus & Bendigo & $1976-86$ & Plants, seed & Drought failure \\
\hline L. luteus & Bendigo & $1976-86$ & Plants & Drought failure \\
\hline Medicago sativa & Top-Galloway & $1983-93$ & Seed & Failed after $1 \mathrm{st}$ yr, cold temperatures \\
\hline M. sativa & Earnscleugh A/S" Flat & lat 1992-93 & Plants & Not identified \\
\hline M. sativa & Wolds & $1968-73$ & Plants, seed? & From KF O'Connor trial, not identified \\
\hline M. varia & Top-Galloway & 1983-93 & Drilled & Seed germination failed \\
\hline Melilotus alba & Top-Galloway & 1983-93 & Drilled & Seed germination failed \\
\hline M. officinalis & Bendigo & 1976-86 & Drilled & Failed after $4-5 \mathrm{yrs}$, poor reseeding \\
\hline M. officinalis & Top-Galloway & 1983-93 & Drilled & Seed germination failed \\
\hline Onobrychis viciifolia & Bendigo & $1976-86$ & Drilled & Seed germination failed \\
\hline O. viciifolia & Bendigo & $1976-86$ & Plants & Drought failure \\
\hline Ononis pusilla & Bendigo & 1976-86 & Plants & Drought failure \\
\hline Trifolium ambiguum & Bendigo & $1976-86$ & Plants & Drought failure \\
\hline T. ambiguum & Otematata & 1978-93 & Plants & Drought stressed, frosting? \\
\hline T. fragiferum & Earnscleugh A/S Flat & at $1992-93$ & Plants & Not identified \\
\hline T. hybridum & Top-Galloway & $1983-93$ & O/S Seed & Good initially, now sparse, requires fertility \\
\hline T. medium & Top-Galloway & 1983-93 & Drilled & Seed germination failed \\
\hline Vicia sativa & Earnscleugh A/S Flat & at $1992-93$ & Plants & Not identified \\
\hline V. sativa & Earnscleugh A/S Shad & ady1992-93 & Plants & Not identified \\
\hline
\end{tabular}

$\pi=$ Accelerated screening plots 
$(131-142)$

Table 5 Legume species at identified sites recording highest vigour ranking (3), sorted (in descending order) by survival and productivity ranking with associated plant height/spread $(\mathrm{cm})$, phenology and comments.

\begin{tabular}{|c|c|c|c|c|c|c|c|c|}
\hline Binomial & Site & Altitude & Survival & Height & Spread & Productivity & Phenology & Comments \\
\hline Astragalus falcatus & Stony Creek & 750 & 3 & 65 & 180 & 3 & SD & $\begin{array}{l}\text { Minor die-back, } \\
\text { drought or late frost }\end{array}$ \\
\hline Dorycnium hirsutum & Mid-Galloway & 500 & 3 & 35 & 50 & 3 & FL, FR- & $\begin{array}{l}\text { Plants and seed } \\
\text { good }\end{array}$ \\
\hline D. pentaphyllum & Stony Creek & 750 & 3 & 45 & 100 & 3 & $\mathrm{FL}, \mathrm{FR}$ & $\begin{array}{l}\text { Plants good, no } \\
\text { seedlings noted }\end{array}$ \\
\hline Lupinus polyphyllus & Glentanner-Lower & 840 & 3 & 45 & 35 & 3 & FL-, FR, SD & $\begin{array}{l}\text { Ex pelleting trial, } \\
\text { numerous } \\
\text { seedlings }\end{array}$ \\
\hline L. polyphyllus & Mt John & 760 & 3 & 35 & 45 & 3 & $\mathrm{FR}, \mathrm{SD}$ & $\begin{array}{l}\text { Dominant legume, } \\
\text { some drought stress }\end{array}$ \\
\hline Melilotus alba & Earnscleugh-Omeo & 270 & 3 & 150 & 60 & 3 & $\mathrm{FL}+$ & $\begin{array}{l}\text { Vigorous, reseeded } \\
\text { from drilling }\end{array}$ \\
\hline Trifolium medium & Cockayne-Plot 12 & 900 & 3 & 30 & 300 & 3 & SD+ & $\begin{array}{l}\text { Tops drought } \\
\text { stressed, also in } \\
\text { Plot } 9 \text { in } 1995\end{array}$ \\
\hline T. medium & Sievwright-Holbrook & 670 & 3 & 40 & $2-300$ & 3 & $\mathrm{SD}+$ & Drought stressed \\
\hline D. pentaphyllum & Otematata & 490 & 3 & 50 & 100 & 2 & V & $\begin{array}{l}\text { Some reseeding, } \\
\text { cold and drought } \\
\text { tolerant }\end{array}$ \\
\hline M. officinalis & Earnscleugh-Omeo & 270 & 3 & 100 & 50 & 2 & $\mathrm{FL}+$ & $\begin{array}{l}\text { Vigorous, reseeded } \\
\text { from drilling }\end{array}$ \\
\hline T. medium & Mt John & 760 & 3 & 20 & 300 & 2 & V & $\begin{array}{c}\text { Several large } \\
\text { patches ex Mason } \\
\text { trial }\end{array}$ \\
\hline D. pentaphyllum & Earnscleugh-Omeo & 270 & 3 & 40 & 60 & 2 & $\mathrm{FL}+, \mathrm{FR}-$ & $\begin{array}{l}\text { Little reseeding, } \\
\text { but } v \text { cold and } \\
\text { drought tolerant }\end{array}$ \\
\hline Lotus corniculatus & Stony Creek & 750 & 2 & 30 & 60 & 3 & V & $\begin{array}{l}\text { Minor die-back, } \\
\text { frost? }\end{array}$ \\
\hline Lupinus polyphyllus & Sievwright-Holbrook & 670 & 2 & 65 & 55 & 3 & $\mathrm{FR}, \mathrm{SD}$ & $\begin{array}{l}\text { Drought and late } \\
\text { frost stress } \\
\text { showing }\end{array}$ \\
\hline L. polyphyllus & Glentanner-Scotts Nth & h1250 & 2 & 40 & 35 & 3 & SD- & $\begin{array}{l}\text { OK, ex 1970s } \\
\text { seeding }\end{array}$ \\
\hline T. pratense & Sievwright-Holbrook & 670 & 2 & 45 & 65 & 3 & FL-, SD+ & $\begin{array}{l}\text { Minor drought } \\
\text { stress }\end{array}$ \\
\hline A. falcatus & Mid-Galloway & 500 & 2 & 25 & 55 & 2 & FR-, SD & $\begin{array}{l}\text { Plants OK, seed } \\
\text { germination failed }\end{array}$ \\
\hline D. hirsutum & Otematata & 490 & 2 & 70 & 120 & 2 & SD & $\begin{array}{l}\text { Reseeding, plants } \\
\text { dying but cold/ } \\
\text { drought tolerant }\end{array}$ \\
\hline Lotus corniculatus & Glentanner-Scotts Nth & h1250 & 2 & 25 & 20 & 2 & FL- & $\begin{array}{l}\text { Many seedlings in } \\
\text { SW corner of plot }\end{array}$ \\
\hline L. corniculatus & Otematata & 490 & 2 & 45 & 80 & 2 & V & $\begin{array}{l}\text { Some drought } \\
\text { stress, leaf yellowing }\end{array}$ \\
\hline D. pentaphyllum & Bendigo & 390 & 2 & 35 & 50 & 2 & FL, FR & Generally healthy \\
\hline Medicago sativa & Cockayne-Plot 7 & 600 & 1 & 45 & 30 & 3 & $\mathrm{FL}$ & $\begin{array}{l}\text { Few plants, } \\
\text { dominated by tall } \\
\text { oat grass }\end{array}$ \\
\hline L. pedunculatus & Glentanner-Scotts Nth & h1250 & 1 & 30 & 25 & 2 & $\mathrm{FL}+$ & $\begin{array}{l}\text { Patch in NE } \\
\text { corner of plot }\end{array}$ \\
\hline
\end{tabular}

Phenology: $\mathrm{V}=$ vegetative, $\mathrm{FL}=$ flowering, $\mathrm{FR}=$ fruiting, $\mathrm{SD}=$ seeding. $+/-=$ more or less than average. 
Legume genera that recorded a moderate survival ranking ('2') included Trifolium with seven species, Astragalus (four spp), Coronilla (one spp), Dorycnium (three spp), Lotus (two spp) and Lupinus (two spp).

Other surviving species only recorded a low-ranking (1): Astragalus (four spp), Coronilla (one spp), Dorycnium (one spp), Lotus (two spp), Medicago (two spp), Trifolium (six spp). Species known to have failed (11 genera and $22 \mathrm{spp}$ ) are outlined in Table 4. These failed to survive or thrive for various reasons, presumably related to site adaptability. Where known, the reasons recorded were usually 'germination failure', 'drought failure' or 'frost failure'. Although all legumes in open plots showed evidence of grazing, some more recent than others, grazing was not commonly cited as a reason for failure.

\section{Legume vigour}

Plants with the highest (' 3 ') vigour ranking recorded a ' 3 ' survival ranking at more than $50 \%$ of the sites visited. However, those with the highest survival recorded a ' 3 ' vigour ranking at only $34 \%$ of the sites, thus persistence was not necessarily dependant on vigorous growth. Seven genera (10 spp) recorded the highest vigour ranking (Table 5) and these remained productive even though the assessment was carried out in the latter part of a drought-affected growing season.

High vigour was recorded for various plants across the range of sites visited, so was not restricted to a narrowly defined environmental range despite the relatively dry summer conditions. For instance $T$. medium was recorded as high vigour and productivity at both ungrazed sites (Cockayne and Sievwright trials) and at a recently grazed site (Mt John). Results for $D$. pentaphyllum were similar. It had not been grazed for at least 2-3 months at Stony $\mathrm{Ck}$, Earnscleugh-Omeo or Bendigo, but was recently grazed at Otematata and Earnscleugh-Shepherds Flat.

The majority of surveyed legumes (seven genera and $20 \mathrm{spp}$ ) recorded a moderate vigour (ranked ' 2 '). High survival was noted at $20 \%$ of the sites, with $50 \%$ of them recording moderate survival for the species involved. Trifolium spp were predominant with seven species noted. Others included Astragalus (five spp), Coronilla (one spp), Dorycnium (two spp), Lotus (two spp), Lupinus (one spp) and Medicago (two spp).

Species with a vigour ranking of ' 1 ' included
Astragalus (four spp), Coronilla (one spp), Dorycnium (three spp), Lotus (two spp), Medicago (two spp), Trifolium (nine spp not including T. arvense) and Vicia (one spp).

\section{Altitudinal influence}

Sites covered a range of altitudes. In the sub-300 m 'semi-arid' zone, species with the best ranking were $D$. hirsutum, D. pentaphyllum, M. alba and $M$. officinalis. Astragalus cicer, A. falcatus, A. glycyphyllos, A. mongolicus and L. corniculatus also grew moderately well in this zone.

In the 300-500 $\mathrm{m}$ altitude drought-prone environments, 10 genera ( $17 \mathrm{spp}$ ) were recorded. The best rankings included $D$. hirsutum and $D$. pentaphyllum. Astragalus cicer, L. corniculatus, $M$. sativa, T. ambiguum and T. medium had moderately good rankings.

Legumes were well represented at most sites surveyed within the 'mid'-altitudinal band (500-800 m). Eight genera were present, with about 20 species. Those with best rankings included $A$. falcatus, $D$. pentaphyllum, L. polyphyllus, and T. medium. Lotus corniculatus, Lupinus arborea, T. ambiguum, T. canescens, T. hybridum, T. pratense and T. repens were moderately good.

At the higher altitude Glentanner sites (over $800 \mathrm{~m}$ ) a total of five genera (11 spp) were found. Best species included Lotus corniculatus and L. pedunculatus, Lupinus polyphyllus, T. ambiguum and T. medium. Other legumes growing in that environment exhibited poor-medium vigour and/or survival.

\section{Earnscleugh sunny aspect accelerated screening trial}

Although frost heave and stock ingress had affected all three plots at Earnscleugh-Shepherds Flat, many of the original plantings were still evident. Cattle grazing was evident on $L$. corniculatus and $D$. pentaphyllum, but other species showed little, if any, recent foliage removal. Lotus, Medicago and Dorycnium were among the more palatable species at this site and, despite the recent grazing, generally recorded the greatest plant height.

The sunny aspect plot contained the greatest number of legume specie, and of these, C. varia, $L$. corniculatus, $T$. repens and $T$. pratense had the highest number of plants per replicate (Table 6). Some were probably seedlings from the original plants. 
Coronilla varia, T. ambiguum and T. medium plants appeared to be in original locations, but rhizomatous spread was considerable. Astragalus glycyphyllos, $D$. pentaphyllum and T. canescens plant plots were also readily re-locatable. appeared to provide greater soil surface protection from potential erosion agents and therefore potential for revegetation. Remaining Festuca novaezelandiae stands in the area have a low plant density but, even at just 1 plant $/ \mathrm{m}^{2}$, they provide average groundcover and greater biomass (Table 6), although

Table 6 Legume species survival and growth on the Earnscleugh-Shepherds Flat sunny site after a decade. Mean plant number and Standard Error of Mean (SEM) per replicate ( $0.1 \mathrm{ha})$, mean area and height of plants, and percentage contribution to total plot ground cover are presented. Species are in descending order of ground cover.

\begin{tabular}{|c|c|c|c|c|c|c|}
\hline Binomial & $\begin{array}{l}\text { Plant \# per rep } \\
\text { (SEM) }\end{array}$ & $\begin{array}{l}\text { Mean plant } \\
\text { radius }(\mathrm{mm})\end{array}$ & $\begin{array}{l}\text { Single plant } \\
\text { area }\left(\mathrm{m}^{2}\right)\end{array}$ & $\begin{array}{c}\text { Ground cover } \\
(\% / 0.1 \mathrm{ha})\end{array}$ & $\begin{array}{l}\text { Mean Plant } \\
\text { height }(\mathrm{mm})\end{array}$ & $\begin{array}{c}\text { Fresh plant } \\
\text { volume } \\
\left(\mathrm{m}^{3} / 0.1 \mathrm{ha}\right)\end{array}$ \\
\hline Coronilla varia & $33(3.8)$ & 1100 & 3.8 & 12.6 & 30 & 3.8 \\
\hline Trifolium medium & $11(2.4)$ & 1000 & 3.1 & 3.3 & 30 & 1.0 \\
\hline T. ambiguum 9 & $(0.6)$ & 1100 & 3.8 & 3.2 & 20 & 0.7 \\
\hline T. arvense $20000^{\ddagger}$ & 20 & 0.00 & 2.5 & 60 & 1.5 & \\
\hline T. repens & $50(4.5)$ & 300 & 0.3 & 1.4 & 20 & 0.3 \\
\hline T. red * & $4(1.4)$ & 900 & 2.5 & 1.0 & 25 & 0.25 \\
\hline Lotus corniculatus & $30(2.4)$ & 200 & 0.1 & 0.4 & 200 & 0.8 \\
\hline T. canescens & $9(0.4)$ & 300 & 0.3 & 0.3 & 30 & 0.1 \\
\hline Dorycnium pentaphyllum & $12(2.4)$ & 250 & 0.2 & 0.3 & 150 & 0.4 \\
\hline T. pratense & $22(4.0)$ & 150 & 0.1 & 0.2 & 100 & 0.15 \\
\hline Astragalus glycyphyllos & $3(0.5)$ & 250 & 0.2 & 0.1 & 50 & 0.03 \\
\hline Lotus pedunculatus & $2(0.9)$ & 200 & 0.1 & 0.0 & 150 & 0.03 \\
\hline T. fragiferum & $7(0.6)$ & 100 & 0.03 & 0.0 & 20 & 0.00 \\
\hline Medicago sativa & $2(0.5)$ & 100 & 0.03 & 0.0 & 100 & 0.01 \\
\hline Vicia sativa & $1(0.5)$ & 75 & 0.02 & 0.0 & 30 & 0.00 \\
\hline Festuca novae-zelandiae? & $e^{\pi} .1000$ & 90 & 0.03 & 2.5 & 250 & 6.4 \\
\hline
\end{tabular}

¥ Dominant resident legume, numbers estimated.

* Unidentified rhizomatous red clover.

ॠ Comparative example based on typical local population of 1 plant per $\mathrm{m}^{2}$.

Coronilla varia, T. ambiguum and T. medium had the greatest plant spread (Table 6) but, with a plant height of only 2-3 cm and sparse branching, ground cover during mid-summer was not comprehensive with much bare ground and litter apparent. Potentially, these plants also provided the greatest amount of fresh biomass per replicate, much of which should be acceptable to stock.

Dense, well-foliated plants of $A$. glycyphyllos, $D$. pentaphyllum, $T$. canescens and $T$ fragiferum, although of smaller spread and lower plant density, the latter would typically be of low palatability.

\section{Discussion}

The primary objective of this survey was to determine which legume species survived and grew well over time at a range of hill country trial sites in the central South Island, particularly within drier environments and focussing on species not readily available commercially. In total, about $40 \%$ of the species assessed were established by drilling or oversowing, and the remainder by hand planting. Relating that to 
survival, about $32 \%$ of species that recorded the highest survival ranking (all sites) were established from a direct seeded (drilled or oversown) source. The same was true for about $44 \%$ of species that recorded a moderate survival ranking and $36 \%$ of species that recorded a low survival ranking. Plants of Dorycnium, Lotus, Lupinus and Trifolium species that were established using both direct seeding and hand planting techniques at one or more sites were generally ranked closely for vigour thus, once in the ground, plants grew similarly.

In total, 28 species from 11 genera survived at one or more of the trial areas surveyed. For example, 14 clover species were originally planted at Earnscleugh, of which six survived (Table 6). Based on the parameters assessed (survival, vigour and productivity), approximately bout $30 \%$ of the total surviving plant species were above average in their ranking, several at environmentally disparate sites in both Central Otago and the Mackenzie Basin. The most successful species included $A$. falcatus, C. varia, D. hirsutum and D. pentaphyllum, L. polyphyllus, M. alba and M. officinalis, T. canescens, and T. medium.

Conversely, known establishment failures included 22 species from 11 genera (Table 4) and various reasons were identified for this. In many cases seed failed to germinate, either through poor viability or, more commonly, due to adverse environmental effects (drought, temperature) at, or shortly after, establishment. Of those legumes, certain species cannot be recommended for further investigation where the following environmental constraints apply: $D$. rectum (unseasonal cold temperatures), $D$. suffruticosum (drought), H. coronarium (cold temperatures and drought), Lupinus spp. (drought), Onobrychis viciifolia (drought), Ononis pusilla (drought). Failures that occurred with species like $A$. cicer, Lotus spp., Medicago spp., Melilotus spp., and several Trifolium spp. are more difficult to explain and probably involve factors such as poor establishment, environmental issues, plant maturity and possibly grazing management.

Medium-term revegetation and development for South Island dry country areas within low-moderate (250$550 \mathrm{~m}$ ) altitude environments should be possible using legume selections from the successful species identified in the previous paragraph. For example, Dorycnium species retain forage over winter and can also provide low shelter during lambing if established in specialised forage banks (Wills et al. 1999). They can be drilled and they reseed and survive well even on very dry, exposed sites, but lack of seed is a major drawback. Astragalus, Coronilla and Trifolium species (especially T. medium; Woodman et al. 1992) are probably best applied as a component of seed mixes when tussock grassland development is undertaken, but again, seed is not readily available. Melilotus is a good dryland nurse crop (Wills 1984) and Lupinus has potential for grassland sites receiving rainfall $>550 \mathrm{~mm}$ (Scott et al. 1995); however, seed sources are lacking for both.

Reasons for the poor commercial uptake are diverse and not easy to specify. Establishment and management information relating to many of these legumes has been in the scientific and public arena for a number of years. Considerable interest is still shown by farmers in dryland forage and niche fodder crop development, at least in Central Otago and particularly during drought events. However, that interest remains small scale despite regular and ongoing promotion through field days, monitor farm/ landcare groups, a Sustainable Farming Fund project and Otago Regional Council interest.

Few commercial companies have committed to serious involvement with these legumes, presumably for economic reasons, but their field officers do maintain an interest in them.

Coronilla varia, D. hirsutum, L. polyphyllos and $T$. medium are all considered to have potential for greater development and promotion to increase their availability and use. However, demand may not be large enough to attract investment by seed companies, particularly where site-specific cultivars are developed. Some, like Dorycnium, do have a small supporting market in the forestry industry and opportunities in the international market are a subject for further research.

Where included in the surveyed trials, commercially available 'traditional' legume species like $L$. corniculatus, M. sativa, T. ambiguum, T. pratense and $T$. repens exhibited good survival and average to good vigour and productivity, although some problems were noted in some cases with mature stands degenerating and dying out. Their use under land management systems with medium to high fertility and moisture levels (Scott 2001) in pastoral agricultural systems in New Zealand will continue.

Several of the legumes identified in this survey, including A. falcatus, A. mongolicus and A. cicer, D. 
pentaphyllum, M. alba and M. officinalis and $T$. canescens, show promise in small scale trials but need further evaluation to establish their full potential and most suitable agro-pastoral role. Development of small-scale seed production is needed to better define best site, management and use characteristics for them, and to explore possibilities for commercial production.

This survey did not attempt to review in detail all legume species that have been assessed in previous trials at all times of the growing season. Some of that work is still being documented (Scott et al. in preparation). However, most trials were evaluated after environmental, fertility and management conditions had long since reverted to levels consistent with 'normal' farming practice. Much is already known about the management of the persistent legumes discussed here and basic information is readily available to farmers (MRDC 1992, AgResearch 1995). With the exception of the few non-grazed plots included in this survey, grazing management was too variable to comment on with any confidence, other than to say most trials had been grazed on a similar basis to surrounding paddocks for a number of years. The objective was to identify legume persistence after an extended period of reasonably conventional grazing management and, by then applying existing management knowledge if and when these plants are used, there is a good chance they may be successfully utilised under practical farming situations.

The information collected on plant survival and growth during what was a yet another very dry growing season is a valuable indicator of those legume species with potential to help revegetate or develop dryland farming areas not ideally suited to improvement with commercial seed varieties. However, as only small quantities of seed are available, farmers are advised to keep in close contact with the equally scarce, surviving researchers to maximise opportunities for dryland legume development utilising these species.

\section{ACKNOWLEDGEMENTS}

We thank runholders in Central Otago and the Mackenzie Basin for their co-operation, AgResearch staff for assisting with text reviewing and R. Littlejohn for assistance with data analysis.

\section{REFERENCES}

AgResearch. 1995. The Grasslands range of forage and conservation plants. AgResearch Grasslands.
$76 \mathrm{pp}$.

Chapman, H.P.; Keoghan, J.M.; Cossens, C.G.; Allan, B.E.; Littlejohn, R.P. 1989. Promising pasture species for drought-prone slopes in the South Island high country. Proceedings of the Agronomy Society of New Zealand 19: 77-84.

Douglas, G.B.; Wills, B.J.; Pryor, H.N.; Foote, A.G.; Trainor, K.D. 1996. Establishment of perennial legume species in drought-prone, North and South Island sites. Proceedings of the New Zealand Grassland Association 58: 253-257.

Fraser, W. 1992. Accelerated identification of valuable new forage plant introductions in Central Otago (Earnscleugh Station). AgResearch Project proposal IY 851/01, unpublished report.

Haydock, K.P.; Shaw, N.H. 1975. The comparative yield method for estimating dry matter yield of pasture. Australian Journal of Experimental Agriculture 15: 663-670.

MRDC Field Day Publications. 1992. Belfield, Hakataramea Valley, Future options for dryland pastures, December 1991; Ardlui, Middlemarch. Future options for dryland pastures, March 1992; North Otago, Securing the future of dryland pastoral farming in the east coast downlands, March 1992; Glenfoyle, Central Otago, Partners searching for better dryland pastures, May 1992; Earnscleugh, Central Otago, Towards 2000, December 1992.

Scott, D. 2001. Sustainability of NZ high country pastures under contrasting development inputs. 7. Environmental gradients, plant species selection, and diversity. New Zealand Journal of Agricultural Research 44: 59-90.

Scott, D.; Fraser, W. J.; Keoghan, J. M. Plant introduction trials: Evaluations in the high country. (in preparation).

Scott, D.; Maunsell, L. A.; Keoghan, J. M.; Allan, B. E.; Lowther, W. L.; Cossens, G. G. 1995. A guide to pastures and pasture species for the New Zealand high country. Palmerston North, New Zealand Grassland Association.

Tomlinson, A.I.; Sansom, J. 1994. Rainfall normals for New Zealand, 1961 to 1990 . NIWA Science and Technology Series No. 3.

Van Kraayenoord, C.W.S.; Hathaway, R.L. 1986. Plant Materials Handbook for Soil Conservation. Water \& Soil Misc. Publication 93, 94: Vols 1 \& 2.

Wills, B.J. 1984. Alternative plant species for revegetation and soil conservation in the tussock grasslands of NZ. Journal of the Tussock Grassland and Mountain Lands Institute, Review 42: 49-58.

Wills, B.J.; Douglas, G.B.; Foote, A.G.; Trainor, K.D. 
1999. Germplasm characterization and palatability of Dorycnium species under New Zealand dryland conditions. Plant Genetic Resources Newsletter, No 120: 8-14.

Woodman, R.F.; Keoghan, J.M.; Allan, B.E. 1992. Pasture species for drought-prone lower slopes in the South Island high country. Proceedings of the New Zealand Grassland Association 54: 115120.

Woodman, R.F.; Keoghan, J.M.; Allan, B.E. 1996. Pasture legumes for drought-prone outwash soils of the southern Mackenzie Basin. Proceedings of the New Zealand Grassland Association 58: 247-252. 


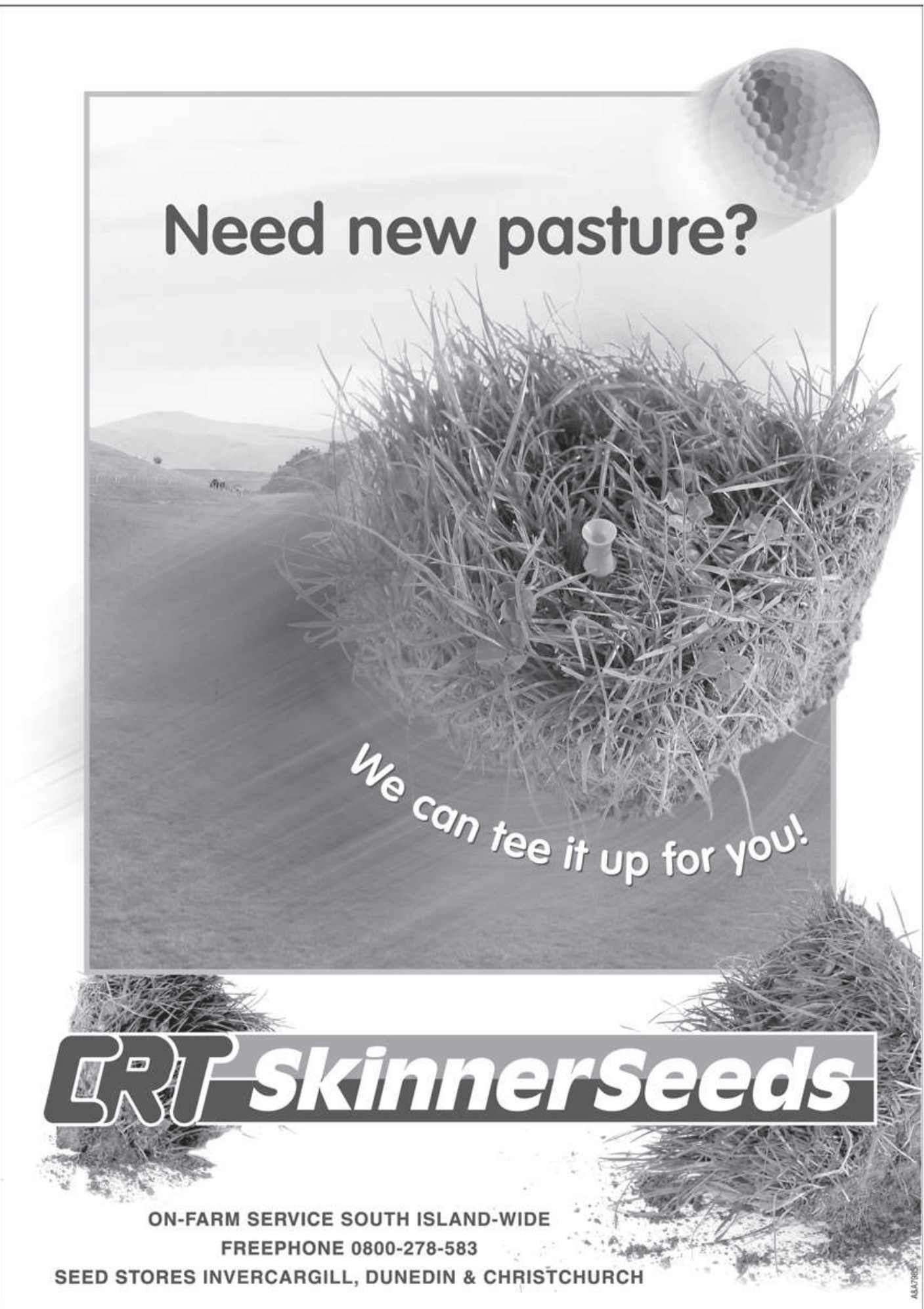

